\title{
Extraction of ionosphere parameters in ionograms using deep learning
}

\author{
Vladimir Mochalov ${ }^{1}$, Anastasia Mochalova \\ Institute of Cosmophysical Research and Radio Wave Propagation of the Far Eastern Branch of \\ Russian Academy of Science, 684034, Kamchatskiy kray, Paratunka, Mirnaya str. 7, Russia
}

\begin{abstract}
Based on a new developed author's method for recognition traces of reflections from different layers of the ionosphere in ionograms, the ionosphere parameters are extracted. The method is based on the use of deep neural networks (DNN). The rules for extracting the ionosphere parameters in ionograms are given. Based on the results obtained by the authors, an intelligent support system for ionogram analysis is being developed.
\end{abstract}

\section{Introduction}

An outstanding feature of the new method proposed by the authors in [1] is the use of deep learning to recognize traces of reflections from different layers of the ionosphere. Deep neural network learning is realized on the basis of etalon markings created by operators. Thus, ionosonde "Parus-A" has been operated at the Institute of Cosmophysical Research and Radio Wave Propagation since August 2015 [2]. It records ionograms every 15 minutes. The operators, interpreting and processing ionograms, make ionogram marking at the beginning of every hour. On average, three out of four ionograms for each hour are unprocessed by the operators. About 45000 ionograms have been processed during the time of "Parus-A" functioning. They are the basis for deep neural network learning. The operators mark ionospheric parameters on the ionograms and detect, when possible, reflection traces from E, F1 and F2 layers of the ionosphere.

The problem of recognition of reflection traces from different ionospheric layers may be referred to the class of object segmentation on images. By the present time, scientists have developed different architectures for deep neural networks, which are used for object segmentation on images (for example, U-Net [3], Mask R-CNN [4], Deep Watershed Transform [5] etc.). Currently, the architecture of deep neural network U-Net has been chosen to detect the reflection traces from different ionospheric layers. In order to recognize the reflection traces from ionospheric E, F1 and F2 layers, we trained a separate deep neural network (DNN) for each layer layer and used black-and-white masks marked by operators (Figures 1,2). We used dice-coefficient loss (DCL) function: $D C L=1-\frac{2|X \cap Y|}{|X|+|Y|}$, where $\mathrm{X}-$ is a set of ionogram layer points marked by operators and $\mathrm{Y}$ - is a set of detected by DNN ionogram layer points. Currently, we have obtained the

1 Corresponding author: vmochalov@ikir.ru 
following results of the DCL function evaluation for a validation dataset when compressing an ionogram to a size of $64 \times 48$ pixels:

a) for DNN for F2 layer recognition DCL is 0.10842 ;

b) for DNN for F1 layer recognition DCL is 0.16890 ;

c) for DNN for E layer recognition DCL is 0.15796 .

The evaluation results of the DCL function for the validation dataset when compressing the ionogram to a size of $192 \times 144$ pixels:

a) for DNN for F2 layer recognition DCL is 0.18859 ;

b) for DNN for F1 layer recognition DCL is 0.22881 ;

c) for DNN for E layer recognition DCL is 0.22209 .
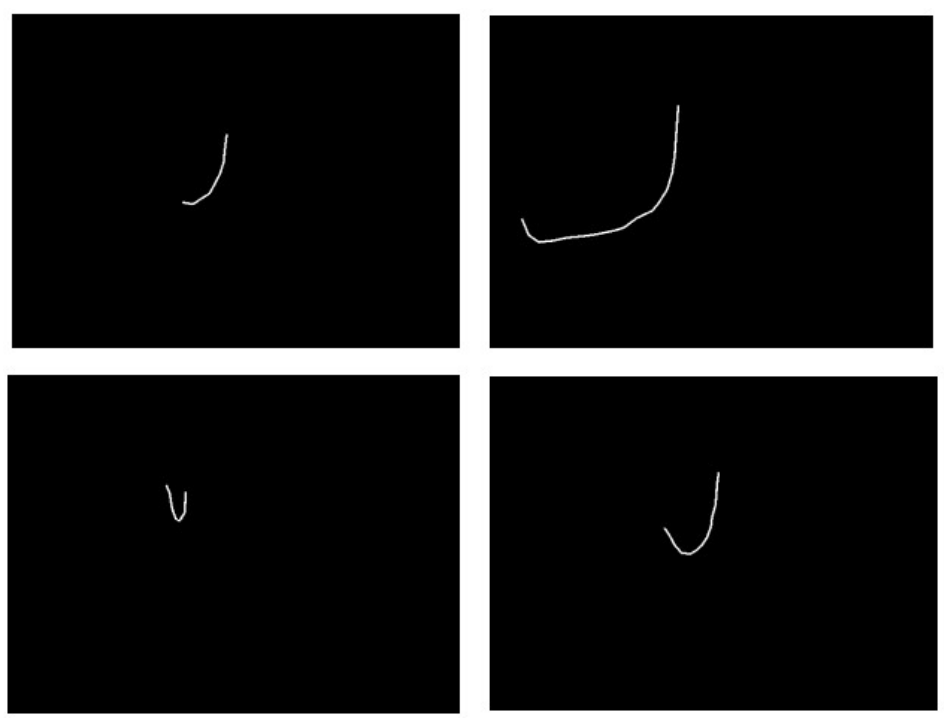

Fig. 1. Examples of black-and-white masks of reflection trace of F2 layer.
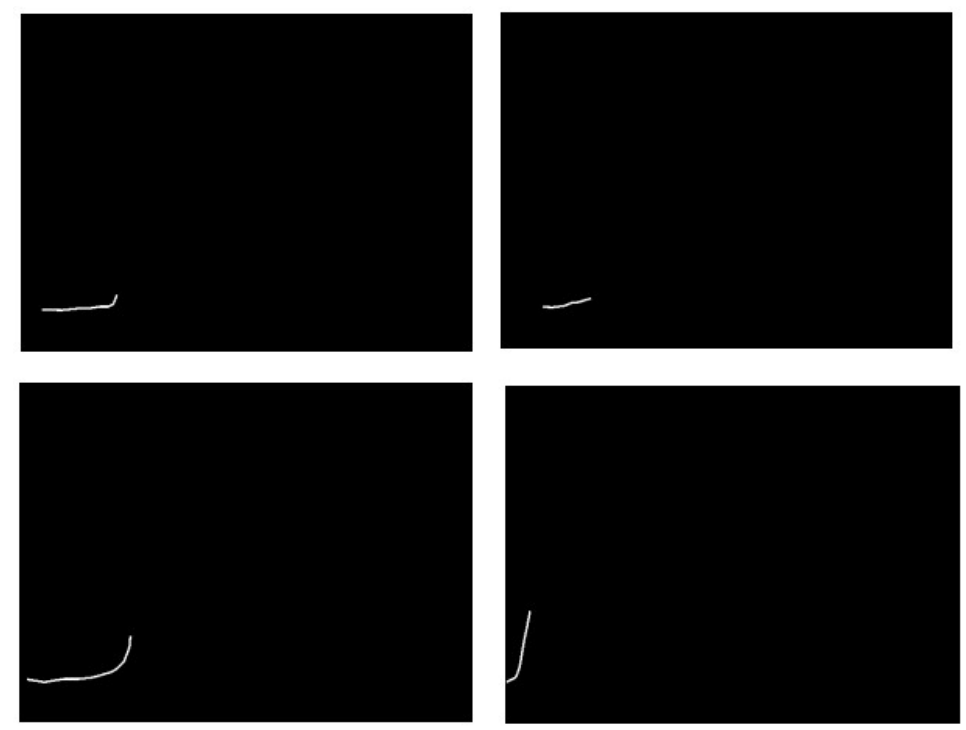

Fig. 2. Examples of black-and-white masks of reflection trace of E layer. 
It should be noted that the total number of training examples for layers F1 and E was smaller compared to layer F2, since at night layers F1 and E disappear and are not labeled by operators. To make a decision on the need to recognize traces of reflection from the F1 and $\mathrm{E}$ layers, the following rule is used: if the solar altitude at the moment of formation of the ionogram at the installation place of the ionosonde is less than the set threshold, then recognition of traces of reflection from the layers is not performed.

\section{Extraction of ionosphere parameters in ionograms}

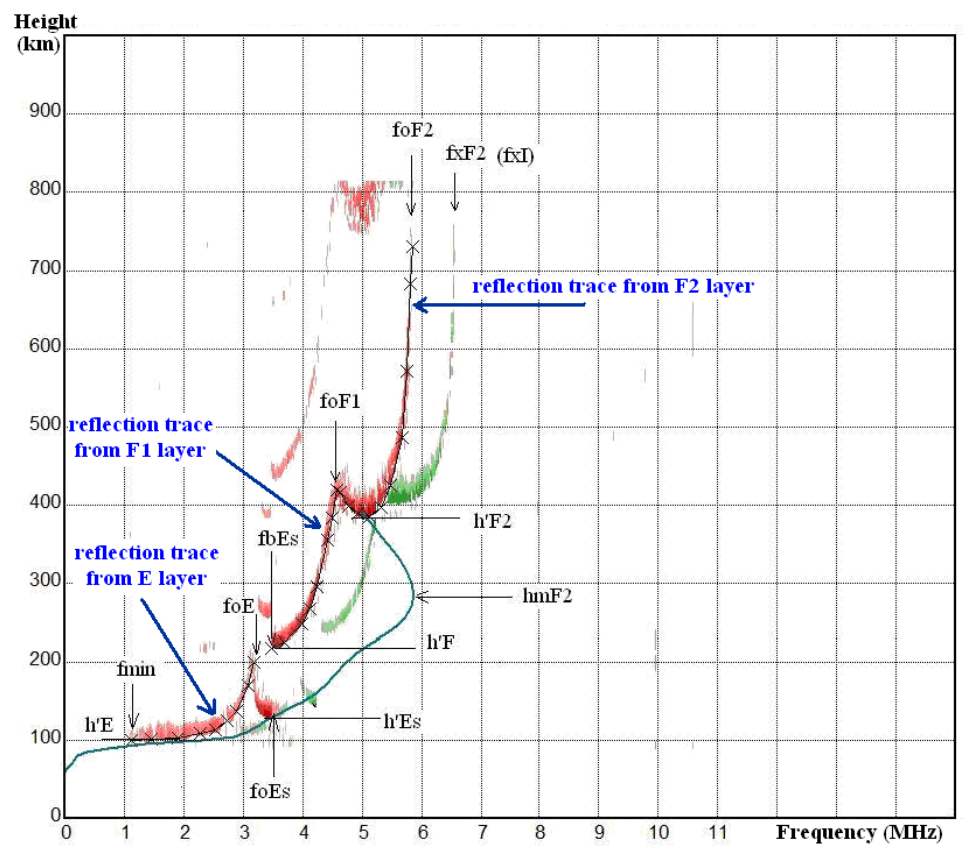

Fig. 3. Example of vertical sounding ionogram with main measurable parameters [6].

Currently, the following basic characteristics are determined by ionograms[6](Figure 3):

- fmin is the lowest frequency at which traces of reflections from the ionosphere are observed on the ionogram;

- foE is the critical frequency of the O-component of the lowest thick layer in region E;

- foF1 is the critical frequency of the O-component reflected from the F1 layer;

- foF2 is the critical frequency of the O-component reflected from the F2 layer;

- $\mathrm{fxF} 2$ is the critical frequency of the X-component reflected from the layer F2;

- fbEs is the screening frequency of the sporadic layer Es, that is, the lowest frequency at which first-order reflections of the O-component from the overlying region are observed;

- foEs is the limiting frequency of the O-component reflected from the Es layer;

- $\mathrm{h}$ 'E is the minimum effective height of the region $\mathrm{E}$;

- h'Es is the minimum effective trace height of reflections from Es used to determine foEs;

- $h^{\prime} F$ is the minimum effective height of the trace of reflections of the O-component from the region $\mathrm{F}$ taken as a whole;

- h'F2 is the minimum effective height of the layer F2.

Using the noted parameters of the ionosphere and / or the traces of reflections from different layers of the ionosphere, the electron concentration profile $\mathrm{N}(\mathrm{h})$ is constructed, 
based on which the heights $\mathrm{hmF} 2, \mathrm{hmF} 1$ and $\mathrm{hmE}$ of the main maximum of the electron concentration of the F2, F1 and E layers are determined, respectively.

Dimensionless characteristics are also determined by ionograms [6]:

- M3000 (F2, F1) is a coefficient showing the ratio of the maximum applicable frequency (MUF) to the critical frequency of a given layer with oblique incidence at a jump distance of $3000 \mathrm{~km}$;

- types of sporadic layers by which reflections from Es are classified. There are eleven special categories by which reflections from sporadic Es layers are classified;

- types of F-scattering - classification of the type of scattered reflections.

The determination of ionospheric characteristics by ionograms is often difficult, and sometimes completely impossible for various reasons (ionospheric or instrumental). Therefore, to explain the difficulties of characterization and classification of phenomena adopted by the international system of letter designations [6].

Based on the selected traces of reflections from different layers of the ionosphere [1], we are able to determine at least the following of the above parameters of the ionosphere: foF2, foF1, foE, h'F2, h'F, h'E. Based on the selected parameters and based on the selected traces of reflections from different layers of the ionosphere, we are able to construct the electron concentration profile $\mathrm{N}(\mathrm{h})$ and then determine the heights $\mathrm{hmF} 2, \mathrm{hmF} 1 \mathrm{and} \mathrm{hmE}$.

Consider the rules for extraction the parameters of the ionosphere.

Rule for extraction foF2 parameter.

The maximum frequency of the selected reflection trace from the F2 layer is the extracted ionosphere parameter foF 2 (an example of the foF 2 extraction is shown in Figure 4).

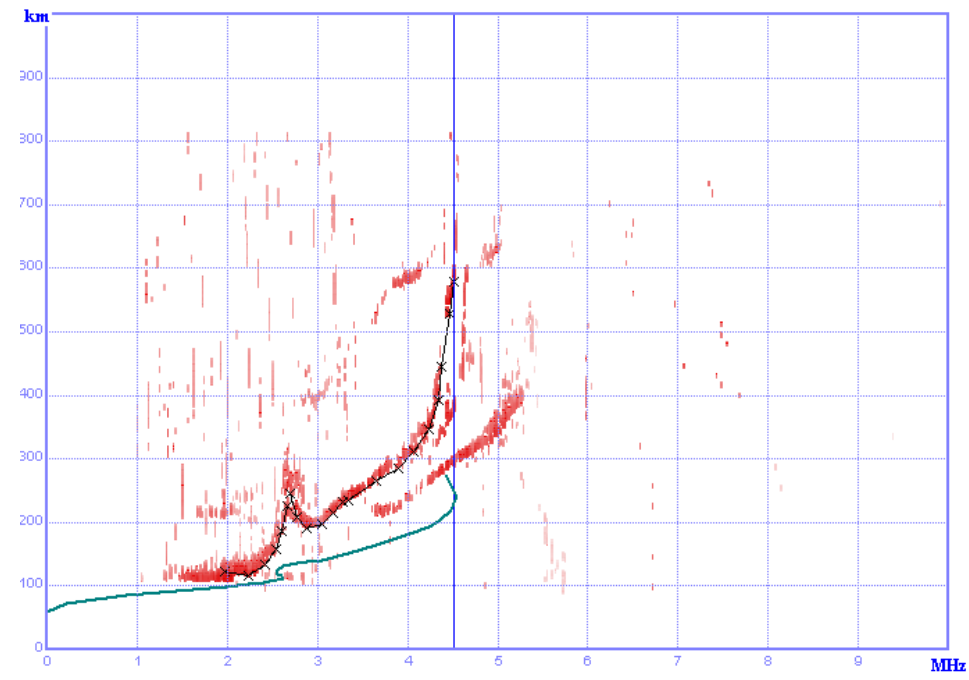

Fig. 4. An example of the extraction of the ionosphere parameter foF2.

\section{$\underline{\text { Rule for extraction foF1 parameter. }}$}

The maximum frequency of the selected reflection trace from the F1 layer is the extracted ionosphere parameter foF1 (an example of the foF1 extraction is shown in Figure 5). 


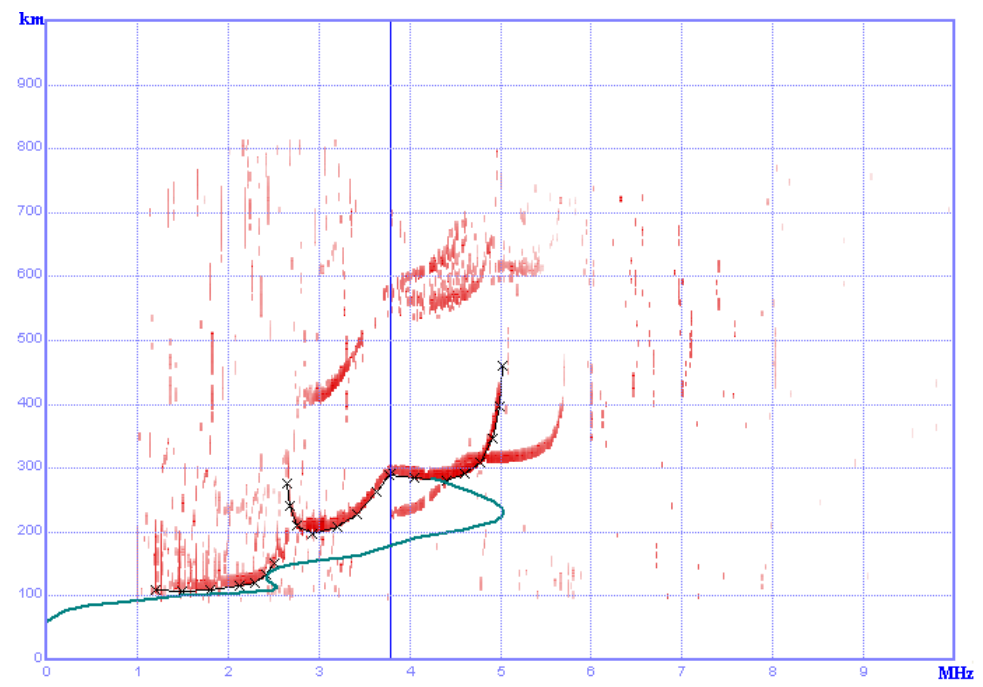

Fig. 5. An example of the extraction of the ionosphere parameter foF1.

Rule for extraction foE parameter.

The maximum frequency of the selected reflection trace from the E layer is the extracted ionosphere parameter foE (an example of the foE extraction is shown in Figure 6).

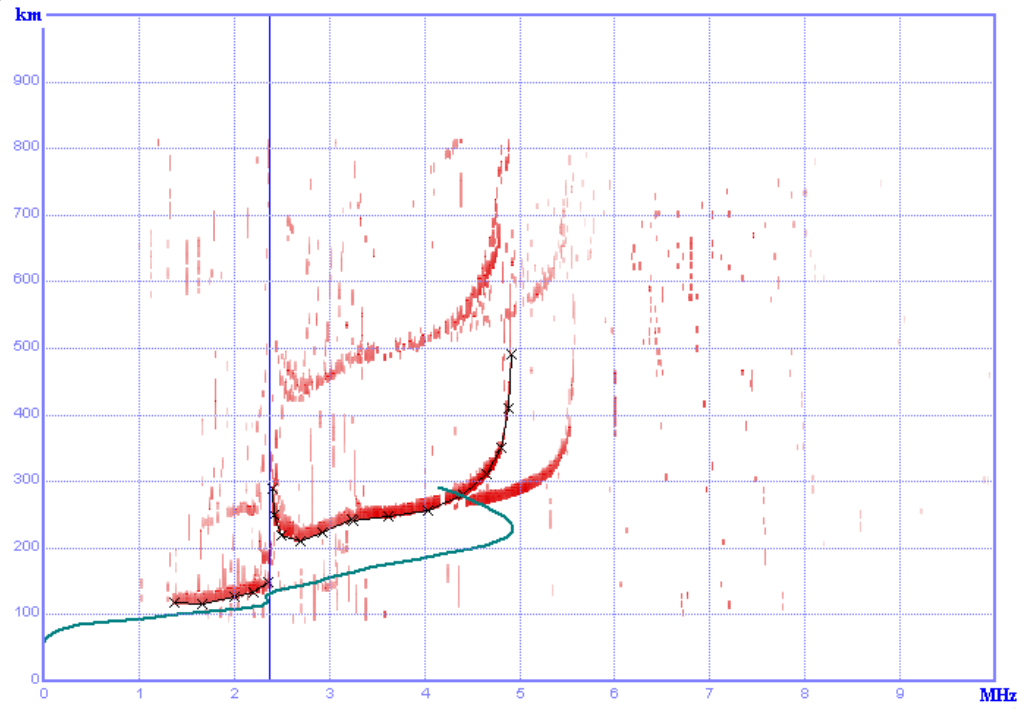

Fig. 6. An example of the extraction of the ionosphere parameter foE.

\section{Rule for extraction $h^{\prime} F 2$ parameter.}

The minimum height of the selected reflection trace from the F2 layer is the extracted ionosphere parameter h'F2 (an example of the h'F2 extraction is shown in Figure 7). 


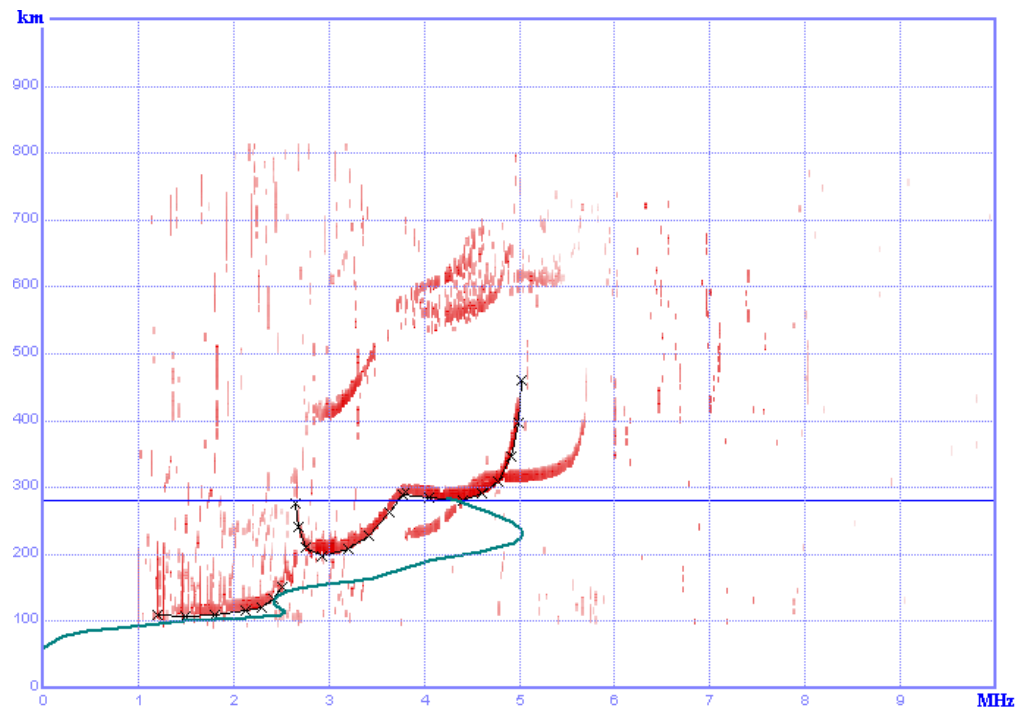

Fig. 7. An example of the extraction of the ionosphere parameter h'F2.

\section{$\underline{\text { Rule for extraction h'F parameter. }}$}

The minimum height of the combined reflection trace from the F layer (created by combining the reflection traces from the F2 and F1 layers) is the extracted ionosphere parameter h'F (an example of the h'F extraction is shown in Figure 8).

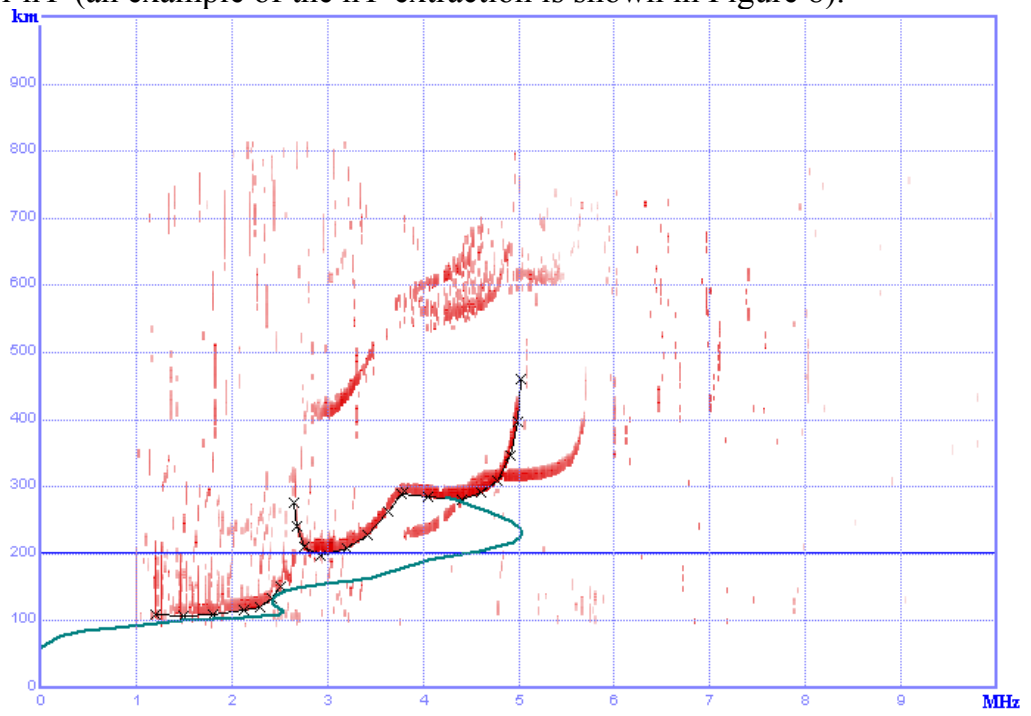

Fig. 8. An example of the extraction of the ionosphere parameter h'F.

\section{Rule for extraction h'E parameter.}

The minimum height of the selected reflection trace from the E layer is the extracted ionosphere parameter $h^{\prime} E$ (an example of the $h^{\prime} E$ extraction is shown in Figure 9). 


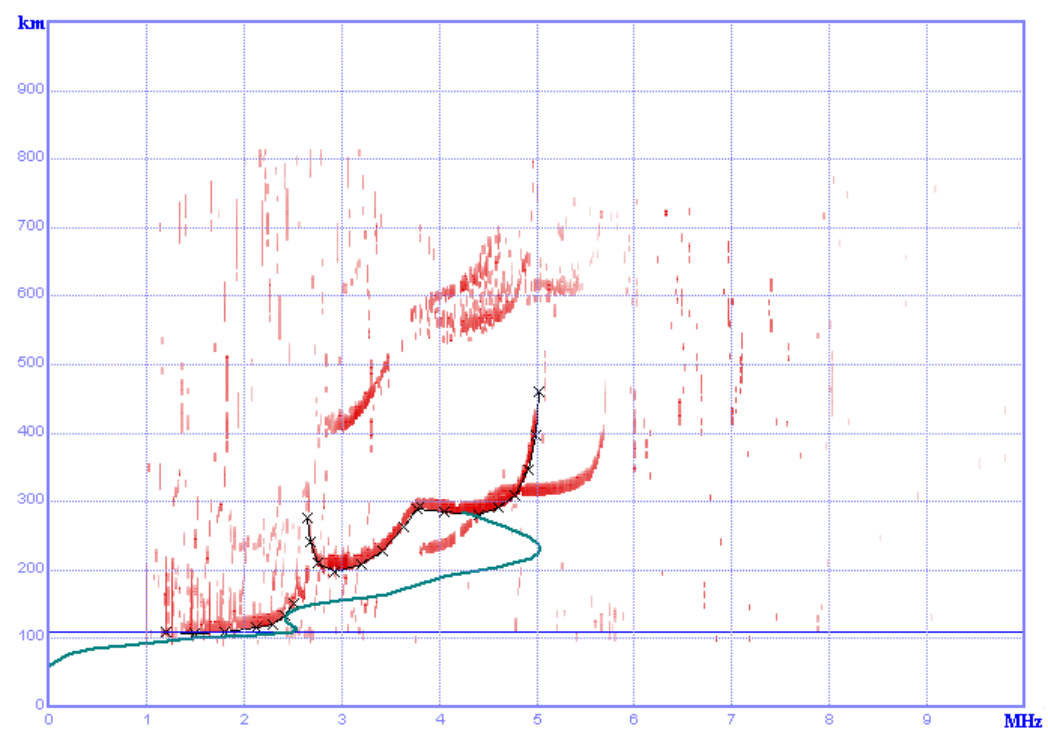

Fig. 9. An example of the extraction of the ionosphere parameter h'E.

\section{Conclusion}

Based on the results obtained by the authors, an intelligent support system for ionogram analysis is being developed [1] (Figure 10). Currently, using the rules given in the work, the following ionosphere parameters are extracted: foF2, foF1, foE, h'F2, h'F, h'E. Based on the selected parameters and based on the selected traces of reflections from different layers of the ionosphere we plan to construct the electron concentration profile $N(h)$ and then determine the heights $\mathrm{hmF} 2, \mathrm{hmF} 1$ and $\mathrm{hmE}$.

\section{Ionogram analysis support system}

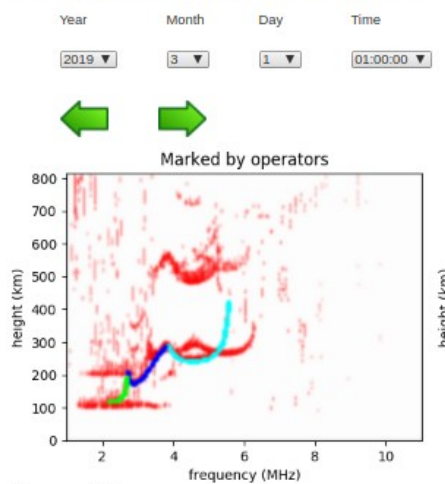

Extracted ionogram parameters:

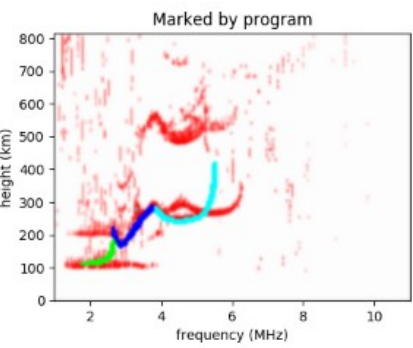

foE $=2.57 \mathrm{MHz}$

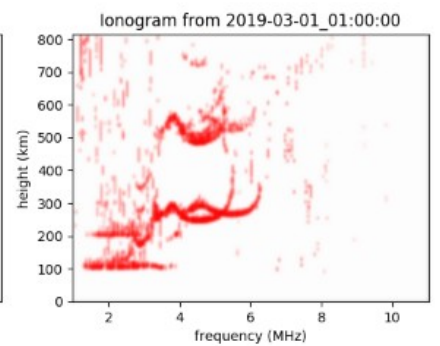

foFl=3.73 MHz h'F $=174.27 \mathrm{~km}$

h'E $=116.92 \mathrm{~km}$

Fig. 10. An example of the extraction of the ionosphere parameter h'E. 


\section{References}

1. V.A. Mochalov, A.V. Mochalova, Proceedings, XXVI Russian open conference RWP, Kazan, 477-479 (2019)

2. G.V. Givishvili, I.V. Krasheninnikov, L.N. Leshchenko, Yu.M. Vlasov, A.V. Kuz'min, Heliogeophysical investigation, 4, 68-74, (2013) (in Russian)

3. O. Ronneberger, P. Fischer, T. Brox, arXiv, https://arxiv.org/abs/1505.04597 (2015)

4. K. He, G. Gkioxari, P. Dollár, R. Girshick, arXiv, https://arxiv.org/abs/1703.06870 (2018)

5. M. Bai, R. Urtasun, arXiv, https://arxiv.org/abs/1611.08303 (2016)

6. Instructions for ionospheric, magnetic and heliophysical observations. Part I. Ionospheric observations. Issue 1, (FGBU “VNIIGMI-MTsD”, Obninsk, 2015) (in Russian). 